\title{
Addressing administrative burden: a primer for submitting comments to the USDA and OLAW
}

\author{
B. Taylor Bennett and Matthew R. Bailey
}

$\mathrm{n}$ the February issue of Lab Animal, we
provided an overview of two opportunities
for the research community to address regulatory burden. One of those was issued by the USDA, titled "Pursuant to Executive Order 13777-Enforcing the Regulatory Reform Agenda," and the final date for comment is July 17,2018 . The second opportunity was the result of language in Section 2034 of the $21^{\text {st }}$ Century Cures Act, which requires that "the Director of National Institutes of Health, in collaboration with the Secretary of Agriculture and the Commissioner of Food and Drugs, shall complete a review of applicable regulations and policies for the care and use of laboratory animals and make revisions, as appropriate, to reduce administrative burden on investigators while maintaining the integrity and credibility of research findings and protection of research animal." The NIH released a notice requesting comments in the Federal Register (Vol. 83, No. 50 / Wednesday, March 14, 2018, p 11221) and posted Notice Number NOT-18-152 on its website. Comments are due by June 12, 2018.

In this commentary we will review the two different processes for submitting comments because, as stated previously, this is a unique opportunity for the regulated community to provide input on changes to regulations, guidelines and policies that could reduce regulatory burden on investigators, which should in turn increase the time available for them to carry out their research.

It should be noted that the current Animal Welfare Regulations and Public Health Service Policy have not been substantially changed since their development following the 1985 amendments to the Animal Welfare Act and the passage of the 1985 Health Research Extension Act. While the regulations and policy have not substantially changed, the responsible agencies have issued guidelines and policies that have affected the regulated community and resulted in administrative burden impacting individual investigators.
Comments submitted in response to the USDA's Request for Information (RFI) can be submitted by mail to the address contained in the Federal Register notice or electronically through the Regulations. gov website (https://www.regulations. gov/document?D=USDA-2017-00020001). The RFI includes four questions the USDA would like commenters to consider, but one is not required to address them individually or at all, and there is no limit to the length of comments. Comments can be written in the online form, or previously developed comments can be uploaded. Some commenters will choose to upload the comments of professional organizations in their entirety while others will simply endorse the comments of a particular organization. Commenters are free to make whatever comments they so choose. All previously submitted comments are available for review in the "Docket Folder."

To submit comments in response to the NIH's request, the simplest way is to go to NOT-OD-18-152 Request for Information (RFI): Animal Care and Use in Research ((https://grants.nih.gov/grants/guide/noticefiles/NOT-OD-18-152.html). The Notice provides the Purpose of the RFI, some Background Information, the Information Requested and How to Submit a Response, which links to the response portal. Unlike the aforementioned Regulations.gov portal, this portal asks commenters for input on specific proposed actions and restricts users to a 1000 word limit for each comment box. There is also a comment box for other approaches not mentioned. OLAW is also asking for feedback on whether five identified tools/resources are or would be helpful in reducing burden on investigators. Unlike the Regulations.gov portal, the NIH portal will not provide access to the comments that have been previously submitted.

The regulatory process in the United States is a participatory process that allows the regulated community to provide their expertise as regulations are being developed, and it has been over thirty years since the current regulations and policies that govern how animal care and use programs operate were proposed. The Final Rule to implement Parts $1 \& 2$ of the Animal Welfare Regulations subsequent to the 1985 amendments to the AWA received 7,857 comments (https://www.nal.usda.gov/awic/ final-rules-animal-welfare-9-cfr-parts-12-and-3), while the more recent USDA RFI published January 26, 2016 entitled "Identifying and Reducing Regulatory Burdens" (https://www.regulations.gov/ docket?D=USDA-2016-0001) received 73 comments, of which only 19 came from the biomedical research community. The current NIH notice lists five reports or surveys which address the issue and/or impact of regulatory/administrative burden on investigators. Reducing that burden would not only allow the investigators to be more productive but allow those responsible for the oversight of the animal care and use program to devote more time and resources to activities that have direct impact on animal welfare.

In this commentary we have described the steps necessary to participate in the regulatory process by making comments on two RFIs which are requesting information on ways to reduce the regulatory/ administrative burden on investigators. In the recent past the research community has failed to take full advantage of this participatory process at the level it did in the late 1980's. That needs to change. It is important that federal agencies requesting this information hear from the regulated community so they fully understand which regulations, guidelines and policies could be revised to reduce administrative burden on investigators "while maintaining the integrity and credibility of research findings and protection of research animals."

B. Taylor Bennett* and Matthew R. Bailey National Association for Biomedical Research, Washington, DC, USA.

*e-mail:btbdvm@yahoo.com

Published online: 23 May 2018 https://doi.org/10.1038/s41684-018-0072-1 